Aim of the study: To characterise expression of mTOR (mammalian target of rapamycin) in childhood B-cell acute lymphoblastic leukaemia (ALL), and to evaluate a possible link between mTOR and clinical characteristics.

Material and methods: The examined group consisted of 21 consecutive patients, aged 1-18 years, diagnosed with B-cell ALL in 2010, and 10 relapsed B-cell ALL patients diagnosed for the first time between 2009 and 2011, who developed relapse before 2014. All subjects were treated in the Department of Paediatric Haematology and Oncology of the Medical University of Warsaw according to the ALL-IC BFM 2002 Protocol. We evaluated mTOR and phospho-mTOR expression by immunohistochemistry using rabbit monoclonal antibodies. Results: mTOR expression was found to be significantly associated with the risk of relapse and was more frequent in ALL recurrence. No significant relationship was detected between mTOR expression and other features of high-risk disease in paediatric ALL. Conclusions: mTOR activity could be considered a high-risk feature in paediatric B-cell ALL. Expression of mTOR kinase is observed remarkably more frequently in disease recurrence than at first diagnosis, indicating higher proliferative and survival potential of leukaemic cells in relapse. Routine analysis of mTOR activity could be performed to select patients that may potentially benefit from mTOR inhibitors (MTI) treatment.

Key words: ALL, childhood, mTOR protein, relapse, prognosis.

Contemp Oncol (Pozn) 2016; 20 (4): 291-296 DOI: $10.5114 /$ wo.2016.61848

\section{Impact of mTOR expression on clinical outcome in paediatric patients with B-cell acute lymphoblastic leukaemia - preliminary report}

Edyta Ulińska ${ }^{1}, K^{2}$ atarzyna Mycko², Elżbieta Sałacińska-Łośs ${ }^{3}$, Agata Pastorczak $^{2}$, Alicja Siwicka ${ }^{1}$, Wojciech Mtynarski ${ }^{2}$, Michat Matysiak ${ }^{1}$

${ }^{1}$ Department of Paediatrics, Haematology, and Oncology, Warsaw Medical University, Poland

2Department of Paediatrics, Oncology, Haematology, and Diabetology, Medical University of tódź, Poland

${ }^{3}$ Laboratory of Pathomorphology, the Medical University of Łódź, Poland

\section{Introduction}

Paediatric acute lymphoblastic leukaemia (ALL) represents a group of biologically and clinically heterogeneous diseases. Although currently $80 \%$ of patients achieve long-term survival, approximately $20 \%$ of children still develop relapse or progression. Therefore, it seems necessary to identify more precise molecular or cytogenetic markers to improve the risk stratification scheme and to develop new targeted biological drugs.

Activity of serine/threonine protein kinase mTOR has recently been considered to play an essential role in the oncogenesis process [1, 2].

The protein kinase TOR functions in two structurally and functionally distinct multiprotein complexes: mammalian target of rapamycin complex 1 (mTORC1) and mammalian target of rapamycin complex 2 (mTORC2) [1, 2]. mTORC1 is a downstream effector of the phosphatidylinositol-3-kinase $(\mathrm{PI} 3 \mathrm{~K})$ /protein kinase B (AKT) pathway, and it becomes active after being phosphorylated at Ser2448 [1, 2]. It phosphorylates eukaryotic initiation factor 4E-binding protein ( $\mathrm{p}-4 \mathrm{E}-\mathrm{BP} 1)$ and $\mathrm{S} 6$ ribosomal protein kinase 1 (S6K1) that are involved in translation and contribute to the control of cell growth, proliferation and survival $[1,2]$. The role of mTORC2 is not fully clear, but it is primarily considered to regulate the cytoskeleton [1, 2]. However, studies have revealed that $\mathrm{mTORC2}$ activates Akt kinase thus stimulating mTORC1 pathway $[1,2]$.

The relation between polymorphisms in $\mathrm{MTOR}$ gene and the risk of ALL among children has been reported [3]. Furthermore, it has already been proven that some recognised genetic aberrations related to adverse clinical prognosis in ALL affect mTOR-mediated signal transduction [4]. One such observation is activation of PI3K/mTOR axis by BCR-ABL1 kinase in leukaemic cells [4]. The efficacy of mTOR inhibitors (MTI) in acute lymphoblastic leukaemia has been evaluated in numerous preclinical studies [5-8] and clinical phase I/II trials, conducted on both adults and children [9-11]. So far, limited data exists on mTOR pathway members expression in childhood ALL blasts $[12,13]$. The influence of mTOR activity on the clinical course and outcome in children with ALL has not been determined either.

\section{Aim of the study}

The aim of our study was to evaluate expression and activity of mTOR kinase in childhood acute B-cell lymphoblastic leukaemia and investigate its association with the clinical characteristics of paediatric B-cell ALL outcome. 
We intended to assess and compare mTOR expression in the first episode and relapse of B-cell ALL in children.

\section{Material and methods}

\section{Patients}

The study enrolled a total of 31 patients (17 males and 14 females) with B-cell ALL, aged 1-18 years (mean 6.9, median 4.7, SD \pm 5 years). The group comprised 10 children diagnosed with B-cell ALL between 2009 and 2011, who developed relapse before 2014, and who were examined to assess and compare mTOR activity in the first episode and relapse of paediatric B-cell ALL. The remaining $21 \mathrm{sub}-$ jects were consecutive patients diagnosed with B-cell ALL in 2010, included to determine if any other clinical characteristics of leukaemia were linked with mTOR expression, except relapse occurrence.

All patients were diagnosed and treated in the Department of Paediatric Haematology and Oncology of the Medical University of Warsaw. The diagnosis was based on bone marrow aspirate examination: cytomorphology assessment, flow cytometry, and genetic analyses. All children were treated according to the ALL-IC BFM 2002 Protocol.

\section{Immunocytochemistry}

mTOR expression was assessed by immunocytochemistry (ICC) staining on bone marrow smears. We examined bone marrow samples from initial ALL diagnosis of all 31
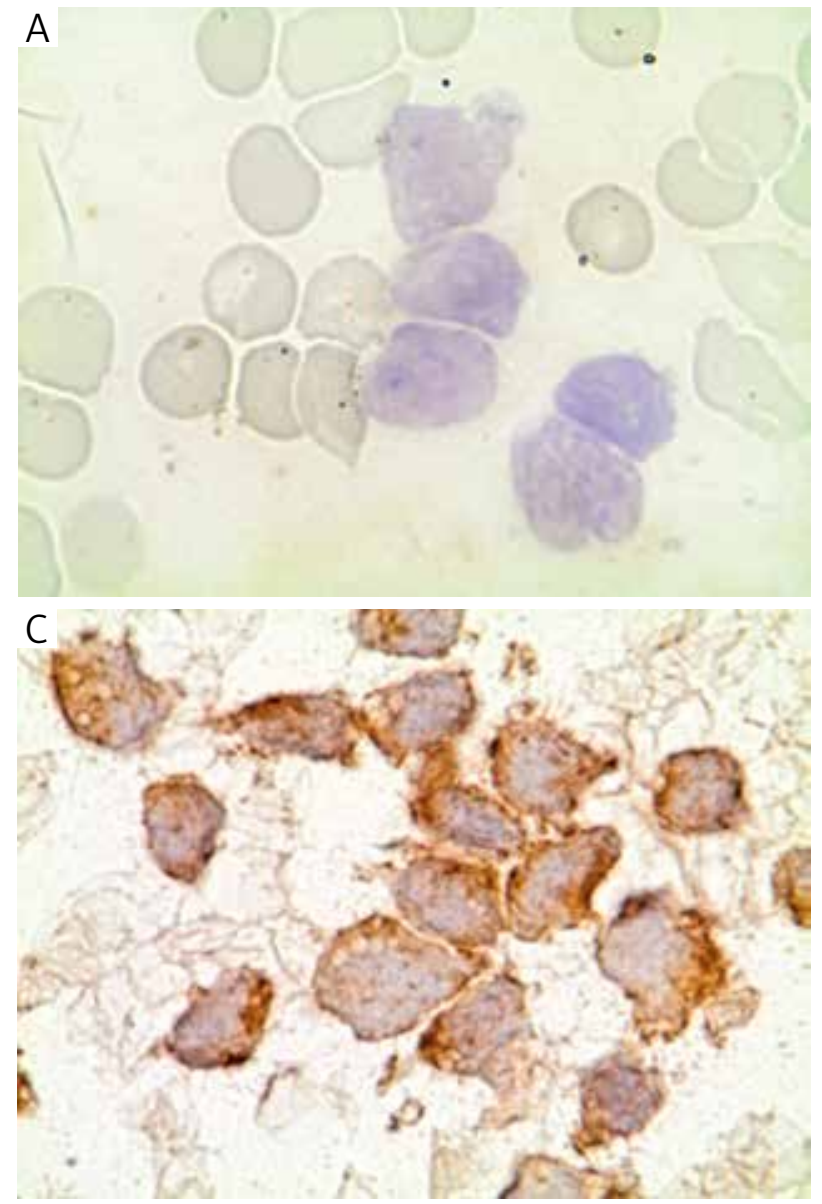

patients and from relapse of 10 patients. One child developed acute myeloid leukaemia (AML) during maintenance chemotherapy of ALL. The myeloblasts of this patient were examined by ICC.

ICC stainings were carried out using primary rabbit monoclonal antibodies anti-mTOR (7C10) and anti-phospho-mTOR (Ser2448) (D9C2) (Cell Signaling, USA). After blocking endogenous peroxidase, bone marrow smears were incubated with diluted primary antibodies (antimTOR 1 : 100; anti-p-mTOR 1 : 50). Immunodetection was performed using a PowerVision detection system (ImmunoLogic, the Netherlands), and visualisation was carried out by DAB (3,3'-diaminobenzidine) (Daco, Denmark, USA). Stainings were evaluated by two cytologists independently, according to the following criteria: (0) no expression, $(1+)$ mild to moderate staining intensity in all blasts or expression visible only in a limited number of blast cells, (2+) strong expression in all blast cells (Fig. 1).

\section{Clinical data}

Clinical data was analysed retrospectively based on patients' medical records and included: age at diagnosis, initial leucocytosis, prednisone response (blast cell count in peripheral blood on day eight of glucocorticosteroids treatment), bone marrow blasts percentage on days 15 and 33 of induction chemotherapy, myeloid antigen co-expression, presence of genetic aberrations associated with poor prognosis (BCR-ABL1 fusion [encoded by the Philadel-

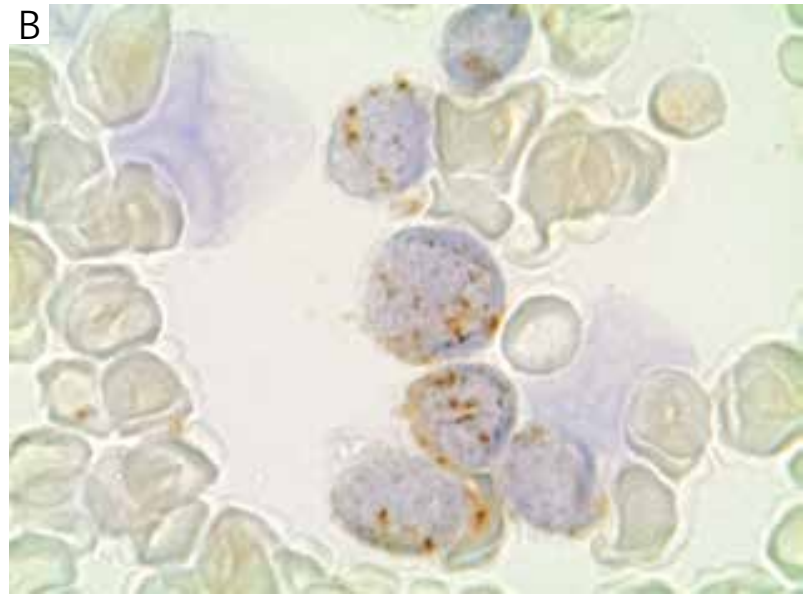

Fig. 1. Representative images of immunohistochemical staining assessment: A) negative staining; B) moderate mTOR expression level; C) high mTOR expression level 
Table 1. Characteristics of patients with positive mTOR expression

\begin{tabular}{|c|c|c|c|c|c|}
\hline \multirow[t]{2}{*}{ Patient's initials } & \multicolumn{2}{|c|}{ I diagnosis } & \multicolumn{2}{|c|}{ Relapse/AML } & \multirow[t]{2}{*}{ Clinical characteristics } \\
\hline & mTOR & p-mTOR & mTOR & p-mTOR & \\
\hline R.K. & + & + & 0 & 0 & HRG \\
\hline S.H. & 0 & 0 & + & ++ & HRG \\
\hline M.M. & 0 & 0 & ++ & ++ & HRG, MLL+ \\
\hline Sz.K. & + & + & 0 (AML) & $+(\mathrm{AML})$ & IRG \\
\hline S.E. & + & + & + & + & IRG \\
\hline W.A. & 0 & $+(P)$ & + & ++ & SRG \\
\hline O.J. & + & + & + & + & HRG, MLL+ \\
\hline M.A. & ++ & ++ & ++ & ++ & IRG, 45XY/46XY \\
\hline T.I. & 0 & $+(\mathrm{P})$ & $+(P)$ & + & SRG \\
\hline H.K. & 0 & + & & & HRG \\
\hline
\end{tabular}

$S R G$ - standard risk group, IRG - intermediate risk group, HRG - high risk group, MLL+ - MLL rearrangement, $P$ - mTOR expression visible only in a limited number of blast cells, $A M L$ - transformation into acute myeloid leukemia

phia chromosome], MLL [Mixed Lineage Leukaemia] rearrangement, hypodiploidy < 45 chromosomes), and disease recurrence.

Bone marrow cells were analysed cytogenetically by standard GTG and CBG banding techniques. Furthermore, FISH (fluorescent in situ hybridisation) was carried out using Vysis MLL Dual Color Break Apart Rearrangement Probe and Vysis BCR/ABL Dual Colour Dual Fusion Translocation Probe to analyse a total of 200 cells.

Myeloid antigen co-expression was detected by multiparametric flow cytometry, using panels of fluorescent-labelled monoclonal antibodies against cell surface antigens including CD13, CD15, and CD33. The criteria for positivity were having more than $20 \%$ positive cells for the markers.

The minimum follow-up period for patients who did not relapse was three years (maximum: 5.5 years), except for two children who died of infectious complications during initial chemotherapy.

\section{Statistical analysis}

The statistical significance of the relations between mTOR expression and clinical parameters was determined using $\chi^{2}$ test and Fisher's exact test. U Mann-Whitney test was performed to assess median age and initial leucocytosis differences between two groups of patients: with detected or excluded mTOR expression. Statistical significance was set at $p<0.05$. Statistical calculations were performed using Statistica 12 (StatSoft, USA).

\section{Results}

\section{mTOR expression at initial diagnosis}

mTOR expression was detected in $8 / 31$ bone marrow samples from the first ALL diagnosis. Positive stainings for the phosphorylated form of mTOR were observed in the case of 5/31 children, who were also mTOR positive. Well-delimited, round, brown cytoplasmic granules were visualised in positive leukaemic cells. In four patients, expression of unphosphorylated mTOR kinase was significantly higher compared to phosphorylated, active form of mTOR both at initial diagnosis and at relapse. However, in other cases p-mTOR and mTOR staining intensity levels were similar. The staining intensity and clinical characteristics of mTOR-positive patients are presented in Table 1.

\section{mTOR expression and clinical outcome}

The group of patients with detected mTOR expression was characterised by older age (mean 8 vs. 6 years, median 5.8 vs. 4.3 years) and higher initial leucocytosis (mean $54.85 \times 10^{9} /$ l vs. $30.98 \times 10^{9} /$; median $15.85 \times 10^{9} /$ I vs. 14.20 $\left.\times 10^{9} / 1\right)$, but the differences were not statistically significant ( $p=0.67$ and $p=0.49$ ). Similarly, we confirmed no statistically significant correlation between the age of diagnosis or initial leucocytosis and p-mTOR expression $(p=0.73$ and $p=0.98)$.

However, we observed a significant relationship between both mTOR and p-mTOR expression and risk of ALL relapse ( $p=0.006, p=0.004)$. Furthermore, we confirmed that there were no statistically significant relations between expression of both forms of mTOR and other features of high-risk disease in paediatric ALL patients with: age $>$ six years $(p=0.69, p=0.92)$, initial leucocytosis $>20 \times 10^{9} / \mathrm{l}(p=1.00, p=1.00)$, poor prednisone response $(p=0.63, p=0.97)$, and bone marrow blasts percentage $>25 \%$ on day $15(p=0.32, p=0.33)$ and $33(p=0.26$, $p=0.66)$. Patients' clinical data and statistical analysis are presented in Table 2.

Mixed lineage leukaemia rearrangement was detected in two patients, and we confirmed mTOR expression at diagnosis in only one of these children. Both of them developed very early mTOR positive relapse. The examined group included one patient with BCR-ABL1 rearrangement, who also developed very early leukaemia recurrence. ICC showed no mTOR-positive staining in his case, neither at the first diagnosis nor at relapse. Cytogenetic analysis revealed no hypodiploidy in the examined group of patients. We did not find any significant relationship between the activity of mTOR or p-mTOR and myeloid antigen co-expression detected by flow cytometry ( $p=0.85, p=0.88)$. One patient with high CD 15+ co-expression developed acute myeloid leukaemia during maintenance therapy of ALL treatment. Lymphoblasts of this patient were mTOR and p-mTOR positive. However, we detected positive stain- 
Table 2. Summary of patients' clinical data and statistical analysis of correlation between mTOR expression and clinical features

\begin{tabular}{|c|c|c|c|c|c|}
\hline & & Number & $\begin{array}{c}\text { mTOR- } \\
\text { p-mTOR- }\end{array}$ & $\begin{array}{c}\text { mTOR+ } \\
\text { p-mTOR+ }\end{array}$ & $p$ \\
\hline Total & & 31 & $\begin{array}{l}23 \\
26\end{array}$ & $\begin{array}{l}8 \\
5\end{array}$ & \\
\hline Sex & $\mathrm{F} / \mathrm{M}$ & $14 / 17$ & $\begin{array}{l}10 / 13 \\
11 / 15\end{array}$ & $\begin{array}{l}4 / 4 \\
3 / 2\end{array}$ & $\begin{array}{l}1.00 \\
0.47\end{array}$ \\
\hline Age (years) & $1-6 />6$ & $18 / 13$ & $\begin{array}{c}14 / 9 \\
15 / 11\end{array}$ & $\begin{array}{l}4 / 4 \\
3 / 2\end{array}$ & $\begin{array}{l}0.69 \\
0.92\end{array}$ \\
\hline WBC & $<20 />20 \times 10^{9} / /$ & $18 / 13$ & $\begin{array}{l}13 / 10 \\
15 / 11\end{array}$ & $\begin{array}{l}5 / 3 \\
3 / 2\end{array}$ & $\begin{array}{l}1.00 \\
0.92\end{array}$ \\
\hline PR & GPR/PPR & $25 / 6$ & $\begin{array}{l}19 / 4 \\
21 / 5\end{array}$ & $\begin{array}{l}6 / 2 \\
4 / 1\end{array}$ & $\begin{array}{l}0.63 \\
0.97\end{array}$ \\
\hline Day 15 of induction & $\mathrm{M} 1 / \mathrm{M} 2 / \mathrm{M} 3$ & $25 / 2 / 4$ & $\begin{array}{l}20 / 1 / 2 \\
22 / 1 / 3\end{array}$ & $\begin{array}{l}5 / 1 / 2 \\
3 / 1 / 1\end{array}$ & $\begin{array}{l}0.32 \\
0.33\end{array}$ \\
\hline Day 33 of induction & $M 1 / M 2 / M 3$ & $30 / 1 / 0$ & $\begin{array}{l}23 / 0 / 0 \\
25 / 1 / 0\end{array}$ & $\begin{array}{l}7 / 1 / 0 \\
5 / 0 / 0\end{array}$ & $\begin{array}{l}0.26 \\
0.66\end{array}$ \\
\hline RG & $\mathrm{SR} / \mathrm{IR} / \mathrm{HR}$ & $8 / 14 / 9$ & $\begin{array}{l}6 / 11 / 6 \\
8 / 11 / 7\end{array}$ & $\begin{array}{l}2 / 3 / 3 \\
0 / 3 / 2\end{array}$ & $\begin{array}{l}0.81 \\
0.35\end{array}$ \\
\hline M.C. & yes/no & $7 / 24$ & $\begin{array}{l}5 / 18 \\
6 / 20\end{array}$ & $\begin{array}{l}2 / 6 \\
1 / 4\end{array}$ & $\begin{array}{l}0.85 \\
0.88\end{array}$ \\
\hline Relapse/AML & yes/no & $11 / 20$ & $\begin{array}{l}4 / 19 \\
6 / 20\end{array}$ & $\begin{array}{l}7 / 1 \\
5 / 0\end{array}$ & $\begin{array}{l}0.006 \\
0.004\end{array}$ \\
\hline
\end{tabular}

F-female; $M$ - male; WBC - initial white blood count; $P R$ - prednisone response on day 8 of treatment; GPR-good prednisone response; PPR - prednisone poor response; $M 1<5 \%$ of blasts in bone marrow; $M 2-5-25 \%$ of blasts in bone marrow; $M 3>25 \%$ of blasts in bone marrow; M.C. - co-expression of myeloid antigens detected in flow cytometry; $A M L$ - transformation into acute myeloid leukaemia

ing only for unphosphorylated form of mTOR in myeloblasts of secondary leukaemia.

\section{Results of ICC staining in relapsed patients}

Seven out of eight mTOR positive patients developed ALL relapse or secondary leukaemia. The remaining patient died of infectious complications during initial chemotherapy and was characterised by unfavourable prognostic factors. All five patients showing positive staining for $\mathrm{p}$-mTOR relapsed. The examined group included two children with mTOR-negative blast cells at the first ALL diagnosis, who had mTOR- and p-mTOR-positive leukaemia cells at relapse.

mTOR expression was found to be more frequent at ALL relapse than at the first diagnosis of the disease (7/10 vs. $8 / 31)$. This relation was revealed with strong statistical significance $(p=0.012)$. The same correlation was confirmed for $p$-mTOR expression (7/10 vs. 5/31, $p=0.001)$.

\section{Discussion}

mTOR pathway plays a key role in cell proliferation, differentiation, growth, and survival [1]. In recent years, extensive research has been conducted on mTOR activity [12, 13] and potential efficacy of MIT in paediatric haematooncological malignancies $[5,6,10]$. Dysregulation of mTOR signalling pathway is considered to be significant in the pathogenesis of childhood ALL and critical for drug resistance development and therapeutic failure [10].

Lizhen Huang et al. reported that genotype variant of mTOR rs2536 is associated with the risk of childhood acute lymphoblastic leukaemia [3].
Despite the fact that many preclinical studies and several clinical trials (phase I/II) were conducted to evaluate MTI efficacy in ALL [9-11], only limited data exists regarding mTOR activity in childhood leukaemic cells $[12,13]$.

Nemes et al. assessed mTOR activity in blasts of 49 paediatric patients, aged $1.8-16.4$ years, with a diagnosis of ALL (41 B-cell ALL, 8 T-cell ALL), treated according to the ALLIC BFM 2002 and 2009 Protocol [12]. They performed ELISA (enzyme-linked immunosorbent assay) measurement of two best characterised mTOR downstream substrates: phosphorylated eukaryotic initiation factor 4E- binding protein ( $p-4 \mathrm{E}-\mathrm{BP} 1$ ) and phosphorylated $\mathrm{S} 6$ ribosomal protein ( $p-S 6)$. The study showed significantly elevated expression of mTOR downstream targets in leukaemic cells, compared to normal lymphocytes, and revealed a statistical relation between relapse occurrence, poor prednisone response (PPR), high risk-group status and mTOR activity in childhood ALL [12].

Correspondingly, our study demonstrates a relation between mTOR expression and higher risk of relapse in paediatric ALL. However, in contrast to the study by Nemes et al., we found no relationship between PPR or higher risk group according to ALL-IC BFM 2002 Protocol and mTOR activity. The small number of patients included in both analyses could contribute to these differences in results. It should be pointed out that the approach to evaluation of mTOR was not the same in the studies. In our research we detected direct mTOR protein expression. The study by Nemes et al. was based on mTOR downstream effectors measurement [12]. Thus, we suggest that analysis of mTOR activity should contain measurement of expression 
of mTOR protein, mTOR substrates, and mTOR transcription.

In our research mTOR expression was found to be significantly more frequent in ALL recurrence compared to the first ALL diagnosis. We believe that this observation indicates the role of mTOR pathway in the mechanism of drug resistance and suggests potential benefit of MTI treatment in this group of patients. In the case of two patients, we observed no mTOR expression at first diagnosis of ALL, but we detected the kinase activity at diagnosis of disease recurrence. On analysing two other children that were initially mTOR positive, we found remarkably amplified reaction of ICC staining in relapse. This observation indicates enhancement of blasts' drug-resistance ability and survival of cells with the highest proliferative and drug-resistance potential [10].

In the last few years, research has been focused on identifying cross-talk and interaction between PI3K/mTOR and best characterised disrupted signalling intracellular pathways in acute leukaemias [4, 14, 15]. Relevant data has been published concerning aberrant PI3K/mTOR signalling transduction in childhood acute lymphoblastic leukaemia characterised by poor prognosis: BCR/ABL1+ [4] and BCR/ABL1-like [13].

mTOR signalling pathway has been found to be upregulated by BCR-ABL1 kinase [6]. Furthermore, the interconnection between PI3K/mTOR and JAK (Janus kinase)/ STAT (signal transducer and activator of transcription) signalling network in CRLF2 (cytokine receptor-like factor 2) rearranged B-cell ALL has been considered [13]. Tasian et al. reported overstimulation of both $\mathrm{PI3K} / \mathrm{mTOR}$ and JAK/ STAT cascades in CRLF2-rearranged ALL [13]. The authors demonstrated reduced expression of Jak/STAT and also $\mathrm{PI} 3 \mathrm{~K} / \mathrm{mTOR}$ pathways' members after JAK inhibitor (ruxolitinib) application. They suggested that overstimulation of both of the signalling cascades mentioned plays a significant role in the pathogenesis of CRLF-2-rearranged ALL, and both JAK and mTOR inhibitors could potentially provide therapeutic benefit to patients with high-risk subtype of ALL [13].

Our study group included only one patient with BCRABL1 mutation, and he was mTOR negative. We presume that confirmation of BCR-ABL1 fusion may not be enough to verify if MTI treatment could be effective in the patient. Thus, mTOR expression assessment could be necessary.

In the last few years in vitro studies have demonstrated that MTI stimulates apoptosis [5] or caspase-independent cell death of childhood ALL blasts [6]. MTOR inhibitors have been found to be effective alone and in combination therapy, indicating a synergistic effect commonly used in ALL treatment cytostatic agents (such as doxorubicin or methotrexate) $[5,7]$ and glucocorticosteroids [8]. Nevertheless, preliminary results of clinical trials on MTI efficacy in refractory ALL in children and adults seem not to be satisfactory [9-11].

The MTI resistance mechanism is not clear [12]. However, the role of an increase in MTORC2 activity and crosstalk between PI3K/mTOR and other intracellular signalling pathways must be considered [12, 16, 17]. Therefore, the effectiveness of dual mTORC1/mTORC2 inhibitors and pan-PI3K/mTORC1/mTORC2 has been extensively studied, and promising experimental results have been published about their effect in leukaemia cells $[12,16,17]$. Simultaneous co-inhibition of several signal transduction pathways may constitute a complimentary approach and is believed to achieve clinically significant antileukaemic effects [17]. Thus future efforts should be directed towards identifying patients who may benefit from the above-mentioned therapies $[12,16,17]$

Based on our findings, we suggest that mTOR activity is associated with increased probability of relapse and could be considered as a high-risk feature in paediatric B-cell ALL. We demonstrated that the importance of mTOR's role in promoting leukaemic cell proliferation and survival differs among paediatric patients with B-cell ALL and is probably more significant in leukaemia recurrences. Therefore, mTOR activity assessment could be considered as a method to select a group of patients characterised by poor prognosis that may potentially benefit from targeted treatment with MTI. The evaluation of mTOR expression in blasts could be performed by immunocytochemistry staining.

Due to some important limitations of our study (i.e. small group of patients and selection bias), further research in this area is necessary. Analysis of mTOR upstream and downstream activity is recommended, not only single member of mTOR signalling pathway assessment. The study will be continued as a multi-centre research project.

\section{The authors declare no conflict of interest.}

\section{References}

1. Hay N, Sonenberg N. Upstream and downstream of mTOR. Genes Dev 2004; 18: 1926-45.

2. Pópulo H, Lopes JM, Soares P. The mTOR signaling pathway in human cancer. Int J Mol Sci 2012; 13: 1886-918.

3. Huang L, Huang J, Wu P, et al. Association of genetic variations in mTOR with risk of childhood acute lymphoblastic leukemia in a Chinese population. Leuk Lymphoma 2012; 53: 947-51.

4. Kharas MG, Janes MR, Scarfone VM, Lilly MB, Knight ZA, Shokat KM, Fruman DA. Ablation of PI3K blocks BCR-ABL leukemogenesis in mice, and a dual PI3K/mTOR inhibitor prevents expansion of human BCR-ABL+ leukemia cells. J Clin Invest 2008; 118: 3038-50.

5. Avellino R, Romano S, Parasole R, Bisogni R, Lamberti A, Poggi V, Venuta S, Romano MF. Rapamycin stimulates apoptosis of childhood acute lymphoblastic leukemia cells. Blood 2005; 106: 1400-6.

6. Baraz R, Cisterne A, Saunders PH, et al. mTOR inhibition by everolimus in childhood acute lymphoblastic leukemia induces caspase-independent cell death. PLoS One 2014; 9: e102494.

7. Teachey DT, Sheen C, Hall J, et al. mTOR inhibitors are synergistic with methotrexate: an effective combination to treat acute lymphoblastic leukemia. Blood 2008; 112: 2020-3.

8. Wei G, Twomey D, Lamb J, et al. Gene expression-based chemical genomics identifies rapamycin as a modulator of MCL1 and glucocorticoid resistance. Cancer Cell 2006; 10: 331-42.

9. Teachey DT, Grupp SA, Brown VI. mTOR inhibitors and their potential role in therapy in leukemia and other haematologic malignancies. Br J Haematol 2009; 145: 569-80.

10. Tasian SK, Teachey DT, Rheingold SR. Targeting the PI3K/mTOR pathway in pediatric hematologic malignancies. Front Oncol 2014; 4: 108.

11. Boumber Y, Thomas DA, Ravandi F, et al. Final report of a phase I/II study of Hyper-CVAD Plus RAD001 (everolimus) in patients with relapsed/refractory acute lymphoblastic leukemia. Blood (ASH Annual Meeting Abstracts) 2012; \#3567. 
12. Nemes K, Sebestyén A, Márk A, et al. Mammalian target of rapamycin (mTOR) activity dependent phospho-protein expression in childhood acute lymphoblastic leukemia (ALL). PLoS One 2013; 8: e59335.

13. Tasian S, Doral M, Borowitz M, et al. Aberrant STAT5 and PI3K/mTOR pathway signaling occurs in human CRLF2-rearranged B-precursor acute lymphoblastic leukemia. Blood 2012; 120: 833-42.

14. Chen W, Drakos E, Grammatikaki I, et al. mTOR signaling is activated by FLT3 kinase and promotes survival of FLT3-mutated acute myeloid leukemia cells. Mol Cancer 2010; 9: 292.

15. Valliyammai N, Nirmala K. Clinical relevance of Notch1 in T-cell acute lymphoblastic leukaemia Int Res J Biological Sci 2013; 2: 66-72.

16. Fransecky L, Mochmann L, Baldus C. Outlook on PI3K/AKT/mTOR inhibition in acute leukemia Mol Cell Ther 2015; $3: 2$.

17. Mendoza M, Er EE, Blenis J. The Ras-ERK and PI3K-mTOR pathways: cross-talk and compensation. Trends Biochem Sci 2011; 36: 320-8.

\section{Address for correspondence}

Edyta Ulińska

Department of Paediatrics, Haematology, and Oncology

Medical University of Warsaw

Marszałkowska 24

00-576 Warsaw, Poland

e-mail: edinge.edyta@gmail.com

Submitted: 3.04 .2015

Accepted: 22.06 .2016 